\title{
Seguimiento del proceso de enseñanza-aprendizaje en la asignatura de transmisión de calor impartida en la Universitat Politècnica de València
}

\author{
Vilariño-Feltrer G*, Campillo-Fernández AJ, Montagud C, Navarro-Peris E, \\ Payá-Herrero J, Sarabia-Escrivà E. \\ Departamento de Termodinámica Aplicada, Universitat Politècnica de València (Valencia, Spain) \\ *Correspondencia: guivifel@upv.es.
}

\begin{abstract}
Resumen
El presente marco educativo universitario ha implicado el paso de una enseñanza centrada en los contenidos a una enseñanza centrada en el alumno y el desarrollo de sus capacidades. En este contexto de adaptación, resulta de vital importancia realizar un seguimiento del proceso de enseñanza-aprendizaje que permita detectar los factores que ayudan o dificultan el proceso de aprendizaje del alumno. En esta contribución se presenta el análisis del proceso de enseñanza-aprendizaje en la asignatura de transmisión de calor, impartida en el segundo año de grados de ingeniería. Se realizó una encuesta el curso 2016/2017 sobre una muestra de 139 alumnos participantes de una población total de 499 matriculados y se ha evaluado las respuestas mediante diferentes herramientas estadísticas para describir, comparar y relacionar los grupos muestrales. Los resultados revelan las características idiosincrásicas de la muestra de estudio y permiten determinar, mediante los 7 items analizados, la consecución de los objetivos de la asignatura, la percepción general del alumnado y la eficacia de los diferentes recursos para el aprendizaje empleados.
\end{abstract}

Palabras clave: Experiencia docente, Transmisión de calor, Ingeniería, Recogida de información, Retroalimentación. 
Seguimiento del proceso de enseñanza-aprendizaje en la asignatura de transmisión de calor impartida en la Universitat Politècnica de València

\section{Introducción}

La puesta en marcha del nuevo marco educativo del Plan Bolonia, ha implicado un rediseño y adaptación de los planes de estudio de las titulaciones de grado y máster. En este contexto de adaptación, resulta necesario redefinir la metodología de enseñanza-aprendizaje tradicionalmente empleada en las aulas pasando de estar centrada únicamente en los contenidos (clase magistral) a centrarse en mayor medida en el alumno y en el desarrollo de sus capacidades tal y como menciona M. de Miguel (2006). Y, como en todo proceso de cambio, resulta de vital importancia realizar un proceso de seguimiento y realimentación que debe considerarse tanto desde el punto de vista del alumno como del profesor.

Desde el punto de vista de los alumnos, es necesaria una pronta retroalimentación para que sean conscientes de lo que saben y lo que no, y puedan actuar en consecuencia con suficiente antelación para poder corregir su actitud a lo largo del proceso de enseñanzaaprendizaje (Ferguson, 2011). Desde el punto de vista del profesor, existe una gran variedad de métodos que puede utilizar para realizar un seguimiento de la docencia y obtener una retroalimentación. Uno de ellos consistiría en la realización de encuestas oficiales de la asignatura, las cuales son útiles para detectar puntos que el profesor debe mejorar en su docencia. Dichas encuestas tienen, en ocasiones, un formato y abordan una serie de dimensiones que resultan demasiado generales, pudiendo estar pasando por alto detalles de la opinión del alumnado sobre aspectos relacionados con las actividades docentes llevadas a cabo en la asignatura, así como la carga de trabajo a la que se han visto sometidos, etc. Es por ello que para la retroalimentación del docente resulta conveniente elaborar otro tipo de encuesta más detallada que permita obtener una información más exhaustiva, completa y, a la que se le puedan aplicar técnicas de análisis sofisticadas, diseñadas para tal fin, como son las herramientas estadísticas (Tian, 2007; Betts et al, 2009).

Este artículo presenta el análisis del proceso de enseñanza-aprendizaje de una asignatura con título 'Transmisión de Calor', la cual se trata de una asignatura obligatoria de 4.5 créditos de segundo curso de grado impartida en tres titulaciones de la Escuela Técnica Superior de Ingenieros Industriales (ETSII) en la Universitat Politècnica de València (UPV): Grado en Ingeniería en Tecnologías Industriales (GITI), Grado en Ingeniería de la Energía (GIE), y Grado en Ingeniería de Organización Industrial (GIOI). Este análisis consiste en el estudio estadístico de los resultados de una encuesta realizada para tal fin durante el curso 2016/2017 sobre una muestra de 139 alumnos participantes de una población total de 499 matriculados. 


\section{Contexto de la asignatura Transmisión de Calor}

Con objeto de poder evaluar de una forma adecuada los resultados obtenidos, se pretende describir dónde se encuentra enmarcada la asignatura dentro de los tres grados bajo estudio y las características diferenciales que pueden presentar cada uno de estos grados desde el punto de vista de la formación inicial con la que se enfrenta el alumno a la asignatura. Resulta conveniente señalar que se partió de la hipótesis de que las diferencias (en calificación) entre grados deberían ser reducidas pues los contenidos y la evaluación en los tres grados se desarrolla de forma conjunta en este y otros contextos de su plan de estudios.

La asignatura de Transmisión de Calor se encuentra ubicada dentro del módulo común a la rama de ingeniería en la materia de termodinámica y mecánica de fluidos en el segundo curso de los tres grados (Guia de estudios ETSII, 2018). En los tres grados se imparte en el segundo cuatrimestre simultáneamente con la asignatura Mecánica de Fluidos y habiendo dado en el cuatrimestre previo la asignatura Termodinámica.

En cuanto a las diferencias en la estructura de los tres grados que pueden afectar al desarrollo de esta asignatura, más allá de la orientación profesional final de cada uno de los grados, en los primeros cursos estos poseen una estructura similar teniendo las mismas materias y asignaturas en los módulos de formación básica y formación común a la rama industrial. Pero es en segundo curso cuando comienzan a aparecer las primeras diferencias entre los grados, más allá de las características idiosincrásicas de la ingeniería, que requieren un análisis muy diferente a otras titulaciones (Dym et al, 2005). GITI y de GIE disponen del módulo de ampliación de la formación básica mientras que GIOI ha sustituido este por el módulo de tecnologías de organización industrial. En el primer módulo es donde los estudiantes reciben la formación básica para la resolución de ecuaciones diferenciales ordinarias, por lo que, para los alumnos de GIOI las asignatura de Transmisión de Calor y en la de Mecánica de Fluidos constituyen su primer contacto con la resolución de este tipo de ecuaciones.

\section{Metodología y alcance de la encuesta}

La encuesta que se utilizó para recabar la opinión de los alumnos de las tres titulaciones que compartían la misma asignatura se componía de 7 preguntas, junto con un espacio final de texto libre para que el encuestado incluyese comentarios de mejora, sugerencia o queja, siendo 7 - 10 min la duración estimada para poder completarla.

Para facilitar la difusión, poder cruzar con las notas de la asignatura y proteger la privacidad de los datos de los encuestados, la encuesta fue anunciada y se debía contestar mediante la herramienta Exámenes de la plataforma de contenidos multimedia PoliformaT, un recurso web de uso conocido, frecuente y necesario para el alumnado, habilitado para 
todas las asignaturas de todos los grados ofertados por la UPV. No obstante, junto con la publicación de la encuesta se envió un mensaje automático al correo institucional de todos los alumnos, instándoles a participar en ella con el objetivo de recabar información y mejorar el proceso de enseñanza-aprendizaje en la asignatura.

La encuesta fue publicada el 30 de junio de 2017, el mismo día en que se publicaron las notas finales de la asignatura, y se les notificó que disponían de un plazo de 1 semana desde el momento de la publicación para contestarla. Con el objetivo de poder cruzar las calificaciones finales de los encuestados con las correspondientes respuestas recabadas en la encuesta, esta no se hizo anónima, si bien el primer tratamiento de datos que se realizó fue la eliminación de datos personales como el nombre y el D.N.I. Todo el análisis, cruce, contraste y representación de datos se realizó con el software estadístico Statgraphics Centurion XVI.

\section{Descripción de la muestra encuestada}

A pesar de las limitaciones de la encuesta, y teniendo en cuenta que el mismo día de la publicación de la encuesta comenzaban las vacaciones de verano para los alumnos, el porcentaje de participación fue bastante razonable (27.86\% Global, 95/317 en GITI, 18/84 en GIE, 26/98 en GIOI). Al analizar la representatividad de la muestra encuestada respecto a las calificaciones finales de todos los alumnos de la asignatura, se llegó a la conclusión de que ni la distribución de la nota final de todos el alumnado ni la de la nota final de los encuestados es normal o gaussiana (Figura 1, y también valores de sesgo y curtosis estandarizados). Además, las distribuciones no resultaron ser comparables ( $\mathrm{p}$-valor = 0.0068 según test W de Mann-Whitney de comparación de medianas).

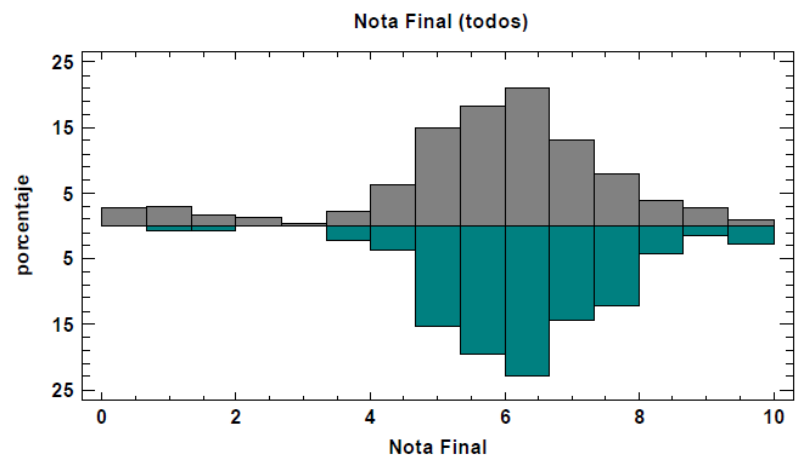

Figura 1. Histograma comparativo de las calificaciones de todos los alumnos de Transmisión de Calor (Nota Final [todos], gris) y de los alumnos de la asignatura que respondieron a la encuesta (Nota Final, verde). 
Cabe destacar, no obstante, que si se comparan las dos variables discriminando los datos para cada titulación (Figura 2), las distribuciones Nota Final (todos) y Nota Final son perfectamente comparables al no encontrarse diferencias estadísticamente significativas.

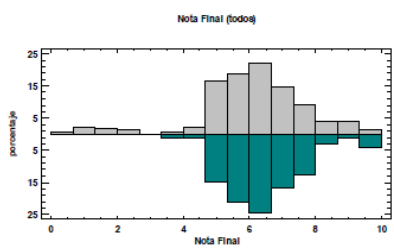

GITI

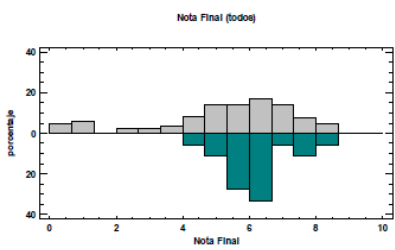

GIE

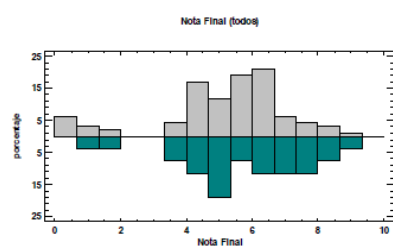

GIOI

\section{Test W ( $\mathrm{p}$-valor:0.089) Test W ( $\mathrm{p}$-valor:0.158) Test W ( $\mathrm{p}$-valor:0.240)}

Figura 2. Histogramas comparativos equivalentes a la Figura 1, con las variables Nota Final (todos) y Nota Final discriminadas por las diferentes titulaciones en las que se imparte la asignatura.

\section{Resultados y discusión}

Tras la descripción general de toda la muestra encuestada, en la presente sección se analizan más en detalle los resultados. En concreto, la Figura 3 muestra la nota final de todos los alumnos matriculados, separada para las 3 titulaciones. Se observa que hay diferencias en las distintas titulaciones, con notas finales de $6.14 \pm 0.88$ para GITI, $5.65 \pm$ 1.12 para GIE y $5.51 \pm 0.98$ para GIOI, expresado como mediana \pm desviación cuartil. Cabe destacar que en GITI, además de ser el grado con mejor nota media, tiene menor dispersión y menor diferencia entre la media y la mediana. Es razonable que en GIOI tengan mayores dificultades para seguir la asignatura, puesto los alumnos no tienen la misma formación previa en asignaturas de matemáticas. Sin embargo, resulta llamativo que en GIE, donde la formación es a priori idéntica a GITI hasta el segundo curso del grado, los resultados sean peores. El hecho de que las notas sean más bajas parece responder más a la población de alumnos con sus características intrínsecas, pues desde hace un par de años se viene observando que tanto en Transmisión de Calor, como en otras asignaturas como Termodinámica Aplicada, donde la evaluación también es la misma, las notas son más bajas en GIE que en GITI. 


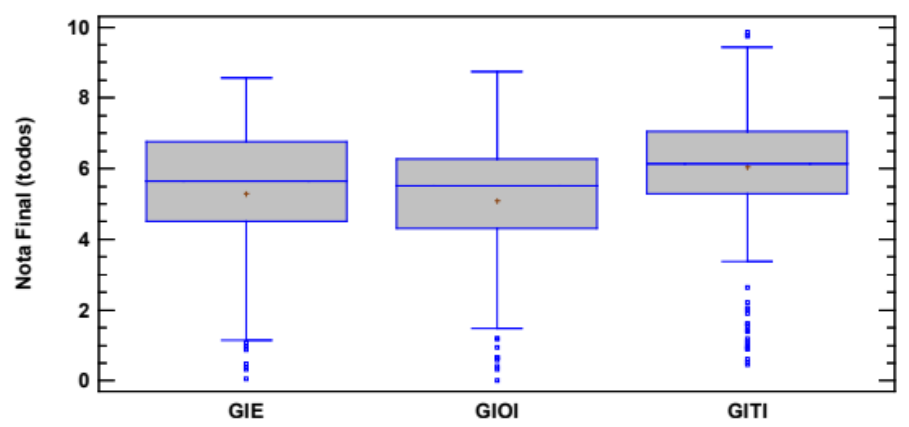

Figura 3. Diagrama de caja y bigotes con la nota final para las 3 titulaciones, para todos los alumnos matriculados.

Este comportamiento heterogéneo entre las diferentes titulaciones ha motivado a que en los sucesivos análisis se presenten, además de los resultados para todos los alumnos (apéndice Total o Global), las respuestas para cada titulación por separado. Cuando la representación e interpretación de los análisis resulta más compleja, se muestran, al menos, los resultados para todo el conjunto y para GITI, el grupo más representativo $(\mathrm{N}=95$ de 139).

Las figuras 4, 5 y 6 muestran algunas de las preguntas exploratorias de la percepción de los alumnos. Resulta interesante destacar las diferentes respuestas que se recogen en función del grado, lo cual indica la percepción que se tiene de la misma según el contexto en el que imparte y la idiosincrasia característica.

La primera pregunta mostrada intenta recoger la percepción que tienen los alumnos sobre el modo en que se ha planteado la asignatura. En este caso, los alumnos de GITI son los que mejor valoran el modo en cómo se ha trabajado en la misma. Se observa que la mitad de ellos entiende que la asignatura ha sido útil en su formación y más del $60 \%$ considera que es interesante, no así en los grados de GIE y GIOI. La asignatura está muy vinculada a conceptos relacionados con GIE y, no obstante, los alumnos de este grado no la encuentran interesante y piensan que ha habido un contenido teórico demasiado alto. El grado de GIOI tampoco tiene unos resultados muy alentadores; sólo un tercio de la muestra piensa que es útil e/o interesante en su formación. El perfil medio del alumno de GIOI no encuentra adecuada esta asignatura en su currículo, ya que entiende que es demasiado técnica. La realidad es que existen otros grado más centrados en administración y gestión empresarial y la diferencia fundamental entre estos y GIOI es precisamente los conocimientos técnicos que los alumnos pueden adquirir en el grado. 


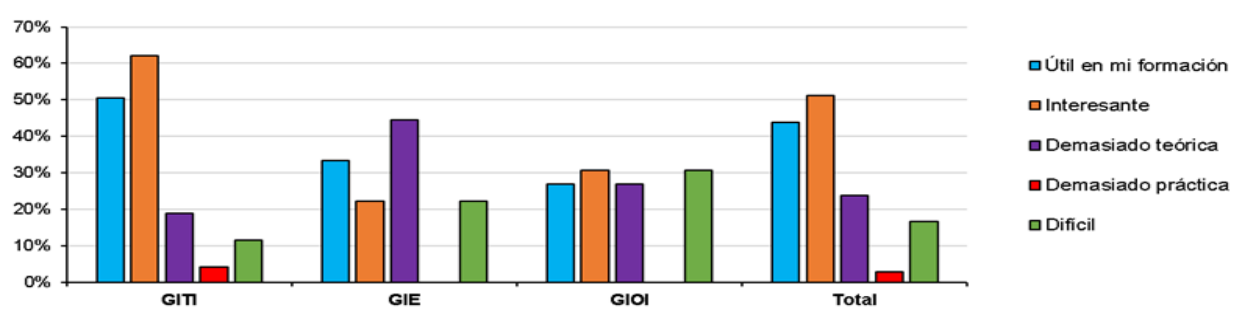

Figura 4. Elección de respuesta múltiple del alumnado ante la afirmación "Considero que la manera en que se ha planteado la asignatura ha resultado...”.

La siguiente pregunta (Figura 5) pretende conocer qué recursos ayudan más a la preparación de la misma. Hay que tener en cuenta que la evaluación de la misma se realiza mediante dos pruebas escritas individuales, y cada una de ellas consta de un test y un problema. Los alumnos de todos los grados consideran necesario el uso de exámenes de años anteriores para baremar el nivel de conocimientos que deben adquirir y con ello afrontar su evaluación. Análogamente sucede con el conjunto de problemas que se aporta para la asignatura. Destacar que los alumnos de GIE no han valorado de forma satisfactoria las clases teóricas de la asignatura, lo que obliga a formular mejoras en esta dirección orientadas a este grupo.

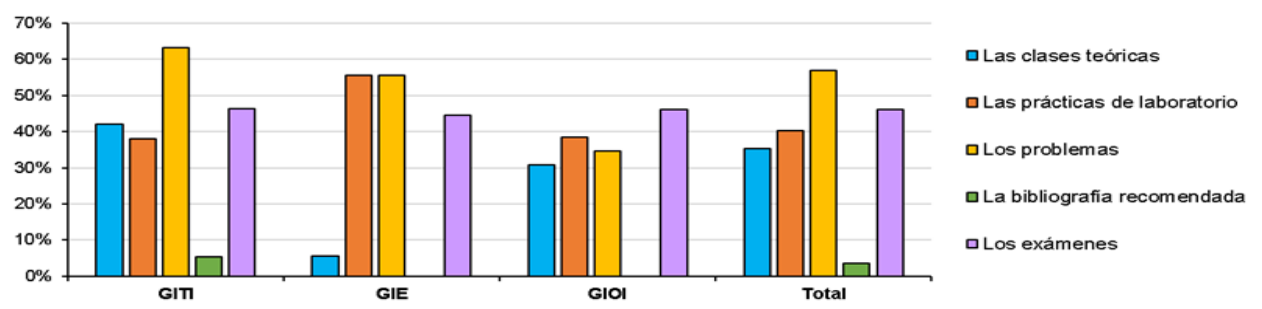

Figura 5. Elección de respuesta múltiple del alumnado ante la pregunta “QQué parte(s) de la asignatura te ha(n) ayudado más a consolidar los conocimientos?”.

La figura 6 muestra qué recursos didácticos consideraría más interesantes el alumno para mejorar el aprendizaje de la asignatura. En este caso, aunque los valores difieren según el grado, existe una tendencia común que es un aumento de clases prácticas y de puestas en común de dudas para mejorar la asignatura, revelando el enfoque que le dan los alumnos a las clases. Para ellos, las clases deben ser un entrenamiento para la evaluación de la asignatura. No valoran los contenidos teóricos porque en la evaluación de la misma estos casi no aparecen de forma explícita. 
Seguimiento del proceso de enseñanza-aprendizaje en la asignatura de transmisión de calor impartida en la Universitat Politècnica de València

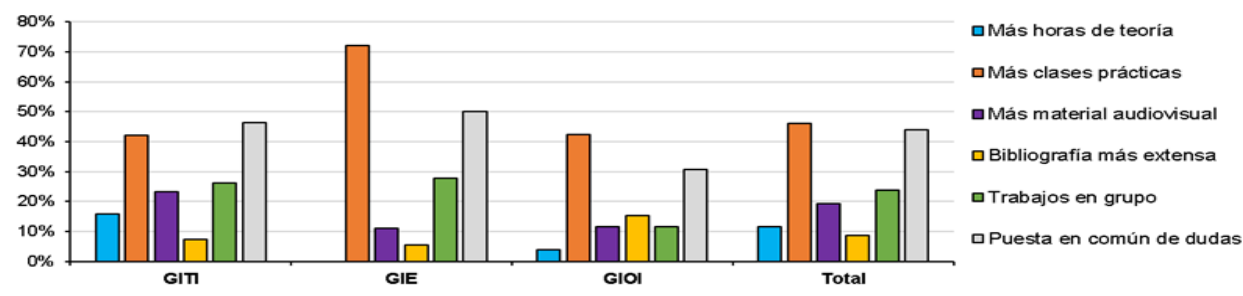

Figura 6. Elección de respuesta múltiple del alumnado ante la pregunta "Desde tu punto de vista, ¿qué recursos didácticos mejorarian el aprendizaje de la transmisión de calor?".

Por otro lado, se ha contrastado la variable Nota Final con las respuestas a otras preguntas formuladas, por la potencia de los resultados que esto puede revelar. Por ejemplo, se puede observar en la Figura 7 que la asistencia en un porcentaje superior al $80 \%$ de las sesiones teóricas y prácticas tiene una crucial relevancia para obtener calificaciones finales altas $(>7)$. Sin embargo, porcentajes menores de asistencia no implican necesariamente el suspenso, ya que no se observa que la mediana sufra grandes cambios con la asistencia. Cabe destacar que los alumnos/as que indican que han dedicado poco tiempo al trabajo no presencial obtienen resultados que no distan mucho de los que le han dedicado mayor número de horas.
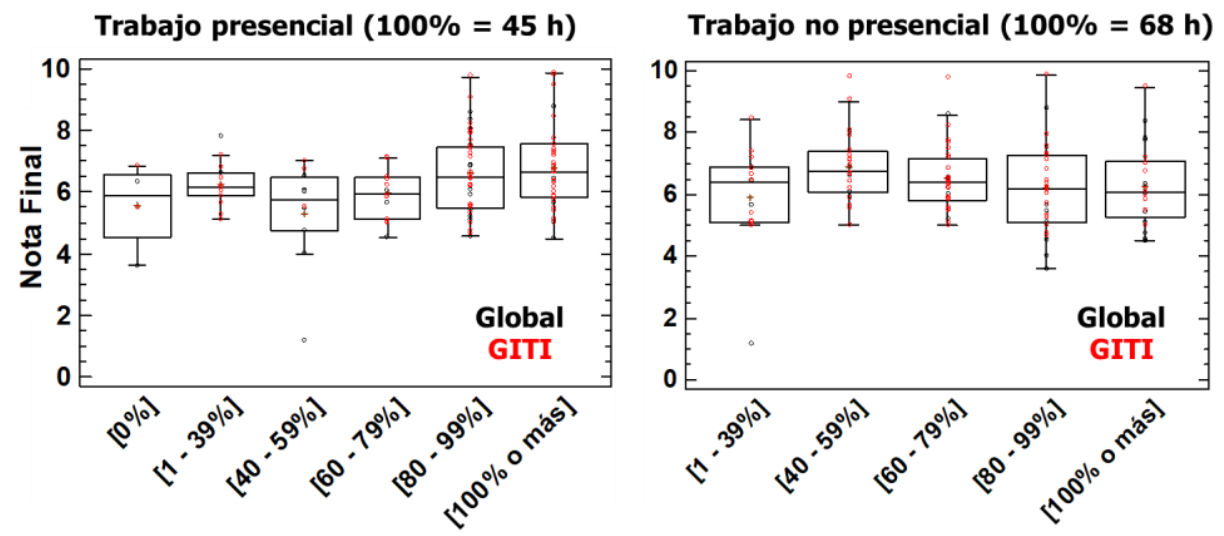

Figura 7. Diagrama de caja y bigotes del trabajo presencial y no presencial y su relación con la calificación final obtenida. Se presentan también los datos individuales. Negro: Global; Rojo: GITI.

Por otra parte, como se puede observar en la Figura 8, acudir a academias externas para preparar la asignatura (independientemente de la asistencia a clase teórica y práctica) no resulta en una mejora sustancial de la calificación obtenida. De hecho, las mejores calificaciones se obtienen por alumnos/as que no asisten a academias tanto en general (diferencia de medianas Global $=0.84$ ) como para GITI (diferencia GITI $=0.67$ ). Este hecho se podría justificar, con cautela, con que la población que asiste a academias puede 
presentar características diferenciales o bien porque el hecho de asistir a academia hace que ese alumnado no asista a clase.
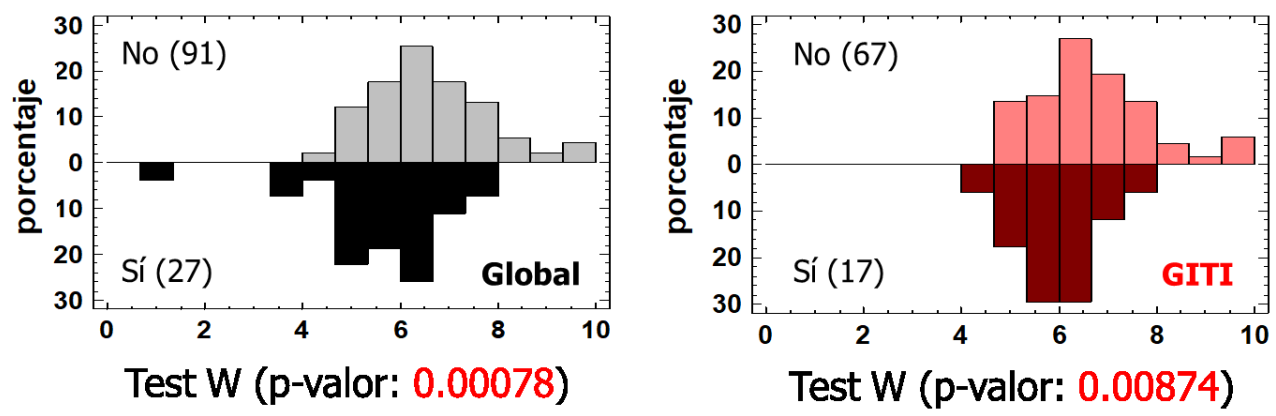

Figura 8. Distribución de Nota Final respecto a si se ha requerido (oscuro) o no (claro) asistir a una academia.

Precisamente, en la figura 9 se observa que los alumnos que más acuden a academias externas tienen menores porcentajes de asistencia a clases teóricas y/o prácticas. Por tanto, acudir a una academia no suele ser un recurso adicional al tiempo de estudio, sino excluyente de éste. A juzgar por el efecto no positivo en la calificación de ir a una academia, resultan preferibles altos porcentajes de dedicación presencial. Es sorprendente que 2 alumnos (GITI) acudieron al $0 \%$ de las clases teóricas y tampoco acudieron a una academia.

Observaciones: 118

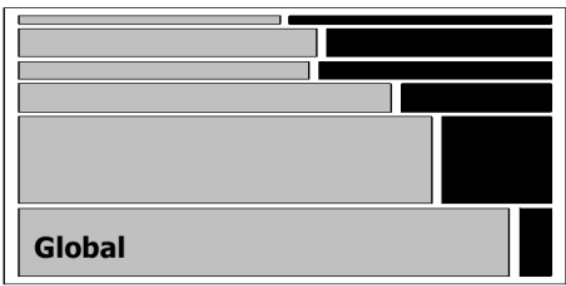

Observaciones: 84

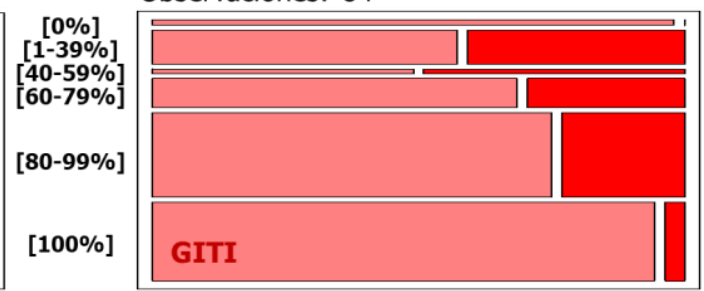

Figura 9. Tablas de relación entre trabajo presencial (\%) y asistencia (oscuro) o no (claro) a academia externa.

Cono análisis final, la Figura 10 muestra la relación entre lo que creen que han aprendido los alumnos (Aprendizaje [subjetivo]) frente a su Nota Final en la asignatura. La tendencia es positiva, aunque con muchísima dispersión, sobre todo en aquellos alumnos que tienen notas comprendidas entre el 4 y el 10. Pese a que la relación no es lineal, resulta interesante destacar que en el grado de GIOI, el coeficiente de correlación $r^{2}$ es mayor que en el resto de titulaciones (0.435). Aunque se evalúa de modo idéntico a tres grados, la forma de puntuar parece representar mejor el nivel de aprendizaje que perciben los alumnos en el grado de GIOI, a diferencia de GIE y GITI, donde la relación con el nivel de aprendizaje subjetivo de los alumnos parece muy débil. Además, la pendiente es muy baja en GIE y 
Seguimiento del proceso de enseñanza-aprendizaje en la asignatura de transmisión de calor impartida en la Universitat Politècnica de València

GITI, lo que refleja que los alumnos sienten poco valorado su aprendizaje en forma de calificación.
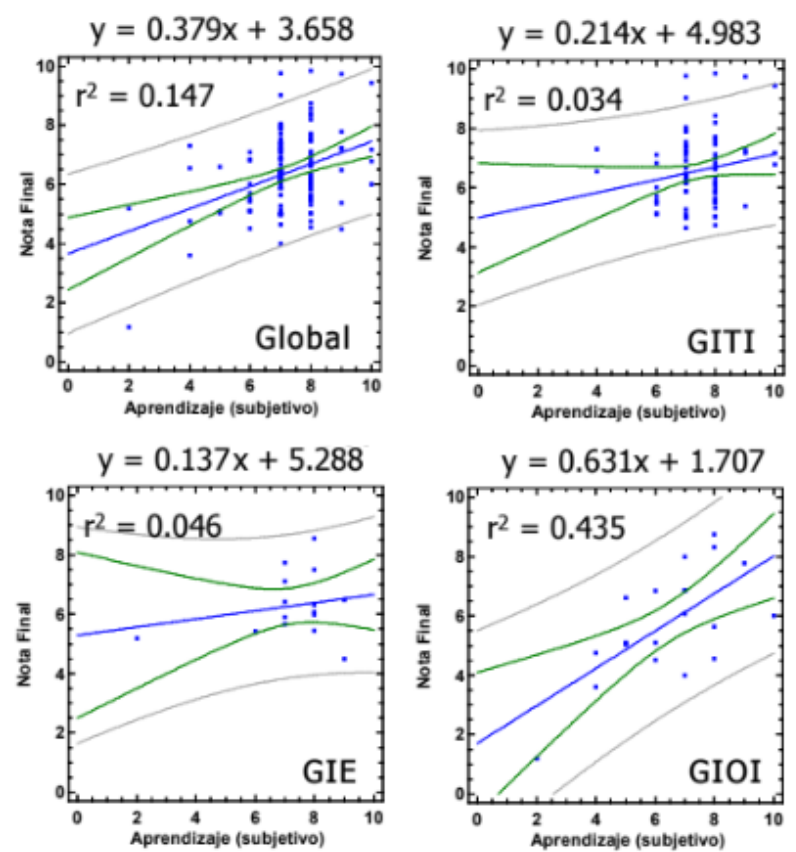

Figura 10. Respuesta ante la pregunta "Indica del 1 (no he aprendido) al 10 (domino el temario) lo que crees haber aprendido en el transcurso de la asignatura” vs Nota Final (numérica).

\section{Conclusiones y modifiaciones a corto plazo}

En cuanto a las tendencias generales acerca de Transmisión de Calor se puede extraer que:

- Los alumnos estudian básicamente realizando problemas y practicando con exámenes de años anteriores. Dan mucha importancia a las prácticas y sesiones grupales de resolución de dudas, pero muy poca al contenido teórico de la asignatura, que sin embargo debería ser una parte relevante en su formación.

- Respecto a la asistencia, las notas más altas en la asignatura las alcanzan siempre alumnos que acuden a más del $80 \%$ de las clases. Los alumnos que menos asisten a clase, en algunos casos se apoyan en academias, pero sus notas no son mejores que el resto.

- No existe una alta correspondencia entre lo que el alumno cree haber aprendido y los resultados de la evaluación. Este punto, unido a que el alumno parece centrar su estudio en la realización de ejercicios prácticos, apuntan a que el alumno no acaba de identificar las habilidades que se espera que desarrolle al realizar esta asignatura. 
No obstante, existen reseñables diferencias entre los distintos grados, a saber:

- En GITI la nota media es más alta, es un grupo más homogéneo y con menos dispersión en las notas finales. Consideran la asignatura más útil e interesante para su formación.

- En GIE y GIOI las notas son algo más bajas que en GITI y con mayor dispersión. Consideran que hay demasiado contenido teórico y poco práctico. Este resultado, que podría hasta cierto punto ser justificable en un estudiante de GIOI, resulta especialmente alarmante en GIE (recordemos, Grado en Ingeniería de la Energía) ya que la transmisión de calor debería constituir un recurso básico en su futuro desempeño profesional.

Gracias a las técnicas estadísticas, se ha obtenido información muy valiosa y se han descubierto ciertos aspectos que se desconocían y cuyo análisis permite desarrollar medidas correctivas o de mejora para los cursos siguientes, para los que a corto plazo se plantea :

- Intentar hacer más hincapié en la importancia de los conocimientos de esta asignatura en el desarrollo posterior del perfil profesional dentro de GIE, relacionándolo en la medida de lo posible con asignaturas de cursos posteriores como Auditorías Energéticas, Frío y Climatización, Centrales Térmicas, etc.

- Alinear el sistema de evaluación empleado con los objetivos que se pretende que el alumno alcance en la asignatura. Para ello se ha propuesto transformar preguntas hacia un carácter más teórico, de razonamiento, para revertir la percepción general.

De cualquier modo, cabe destacar que, a raíz de los resultados, se ha visto el potencial de la encuesta como una herramienta de control y mejora continua de la metodología enseñanzaaprendizaje, pues se piensa seguir realizando en los próximos años, lo que permitirá evaluar su evolución a lo largo de los distintos cursos de impartición de la asignatura.

\section{Referencias}

de Miguel Díaz, M., (2006). Metodologías de Enseñanza y Aprendizaje para el Desarrollo de Competencias. (Orientaciones para el profesorado universitario ante el Espacio Europeo de Educación Superior), 13.

Ferguson, P. (2011). Student perceptions of quality feedback in teacher education. Assessment \& Evaluation in Higher Education, 36, 51-62.

Tian X. (2007). Do assessment methods matter? A sensitivity test. Assessment \& Evaluation in Higher Education, 32, 387-401. 
Seguimiento del proceso de enseñanza-aprendizaje en la asignatura de transmisión de calor impartida en la Universitat Politècnica de València

Betts, L. R., Elder, T. J., Hartley, J., \& Trueman, M. (2009). Does correction for guessing reduce students' performance on multiple-choice examinations? Yes? No? Sometimes? Assessment \& Evaluation in Higher Education, 34, 1-15.

Guia de estudios 2010-2011 de la Escuela Técnica Superior de Ingenieros Industriales de la Universitat Politécnica de València. http://www.etsii.upv.es/docencia/documentos/Guia_Estudios_2010-11_Grados.pdf. (Última visita 05/07/2018).

Dym, C. L., Agogino, A. M., Eris, O., Frey, D. D., \& Leifer, L. J. (2005) Engineering Design Thinking, Teaching, and Learning. Journal of Engineering Education, 94, 103-120. 